\title{
Origin of cosmic rays excess in the Galactic Center
}

\author{
Jouvin L.* \\ APC \\ E-mail: lea.jouvin@apc.in2p3.fr
}

\section{Lemiere A.}

$A P C$

E-mail: alemiereeapc.univ-paris7.fr

\section{Terrier $\mathbf{R}$.}

$A P C$

E-mail: rterrier@apc.in2p3.fr

\begin{abstract}
The center of our Galaxy hosts a Super-Massive Black Hole (SMBH) of about $4 \times 10^{6} \mathrm{M}_{\text {sun }}$. Since it has been argued that the SMBH might accelerate particles up to very high energies, its current and past activity could contribute to the population of Galactic cosmic-rays (CRs). Additionally, the condition in the Galactic Center (GC) are often compared with the one of a starburst system. The high supernovae $(\mathrm{SN})$ rate associated with the strong massive star formation in the region must create a sustained CR injection in the GC via the shocks produced at the time of their explosion.

Indeed, the presence of an excess of very high energy (VHE) cosmic rays in the inner $100 \mathrm{pc}$ of the Galaxy has been revealed in 2006 by the H.E.S.S. collaboration. On very large scale $(\approx 10$ $\mathrm{kpc}$ ), the non-thermal signature of the escaping $\mathrm{GC}$ cosmic rays could have been detected recently as the spectacular "Fermi bubbles". The origin of the CRs over-abundance in the GC still remains mysterious: Is it due to a single impulsive or stationary accelerator at the center or to multiple accelerators filling the region? In order to answer these questions, we build a 3D model of CR injection and propagation with a realistic 3D gas distribution. We then compare with existing data of H.E.S.S.
\end{abstract}

We discuss the CR injection in the region by a spectral and morphology comparison. We place constrains on the SNR rate and on the diffusion parameters.

The 34th International Cosmic Ray Conference,

30 July- 6 August, 2015

The Hague, The Netherlands

\footnotetext{
* Speaker.
} 


\section{Introduction}

The High Energy Stereoscopic System (H.E.S.S.), observing the sky in the very high energy (VHE) domain between $100 \mathrm{GeV}$ and $100 \mathrm{TeV}$, discovered that the center part of our Galaxy presents a diffuse emission localized along the Galactic plane, extending over $2^{\circ}$ in longitude and over $0.3^{\circ}$ in latitude [1]. The close correlation of this emission with the target material in the region suggests that the dominant component of the ridge is due to the interaction of relativistic cosmic rays (CRs) with protons in the ambient medium. The high amount of CRs deduced from this emission is between 3 and 9 times more that the flux measured on Earth with a hard spectrum $(\Gamma \approx 2.3)$. The origin of this over-abundance still remains mysterious.

The SgrA complex located in the inner $10 \mathrm{pc}$ of the Galaxy consists of different structures such as the supernovae (SN) SgrA East (its explosion seems to have occured $10^{-4}$ years ago) as well as the central source $\mathrm{SgrA}^{\star}$, a super massive black hole (SMBH) whose the mass is around $4 \times 10^{6} \mathrm{M}_{\text {sun }}$ [2]. This SMBH is extremely inactive and is fed by the stellar winds in this region. The matter accretion rate at the Bondi radius is around $10^{-5} \mathrm{M}_{\text {sun }} /$ years [3]. In the inner regions, the matter accretion rate is around $10^{-7} \mathrm{M}_{\text {sun }}$ /years [4]. Therefore, [5] deduced the presence of a powerful outflow evacuating the matter. With an actual bolometric luminosity of $10^{36} \mathrm{erg}$, much of the accretion power in the bondi region $\left(10^{39} \mathrm{erg} \mathrm{s}^{-1}\right.$ [5]) is propably used to drive the outflow where CRs acceleration is possible [6].

The inner $150 \mathrm{pc}$ also harbor intense star formation. Around 2\% of the Galaxy's massive star formation occurs at the Center. Knowing that the Galaxy experiences $\sim 2$ SN per century and assuming a regular IMF (initial mass function), one can expect around 0.04 SN per century in the Galactic Center (GC) [7]. The SN rate determined from stellar composition is centered on this value but spread between 0.02 and $0.08 \mathrm{SN}$ per century [7]. Therefore, one of the major issue for modelling the CRs injection by recurrent SNR is the uncertainty concerning the real estimation of the SN rate. This particular region of the Galaxy also contains interstellar $\mathrm{H}_{2}$ gas of about $1.2-6.4 \times 10^{7} \mathrm{M}_{\text {sun }}[8](\sim 10 \%$ of the total molecular mass of the Galaxy) in a rather complex setup of dense molecular clouds $\left(\approx 10^{4} \mathrm{~cm}^{-3}\right)$ called the Central Molecular Zone (CMZ). Around $40 \%$ of the matter in these molecular clouds is spread in four main complexes: Sgr A, Sgr B, Sgr $\mathrm{C}$ and the 1.3 complex (located to the Galactic east from Sgr B2). Many Supernova Remnants (SNR) are visible through their radio and X-ray thermal emission, and Pulsar Wind Nebulae are also numerous in this rich part of the Galaxy.

The total CR energy required to produce the VHE flux of the diffuse emission is around $10^{50}$ erg [1] ( 10\% of the kinetic energy released from a SN explosion [9]). If only one single impulsive injection could explain the energetic of the diffuse emission, we have to account for the additional multiple injection occurring in the region due to the high supernovae rate. In this work, we build a 3D model of CR injection and propagation with a realistic 3D gas distribution. We investigate the scenario where a single source at the GC, stationary (e.g. SgrA ${ }^{\star}$ ) or impulsive (e.g. SgrA East), accelerates the CRs. A recent study [10] has already studied the possible sources of energetic hadrons at the GC considering several scenari of continuous or impulsive injection. However, they analyse these single scenari separately from a stationary steady state model that they consider plausible due to the high SNR rate. Here, in order to build a coherent picture, we will take into account the presence of the high SN rate in the region by modelling these sources by multiple impulsive injection 
throughout the CMZ. We then calculate the resulting $\gamma$-ray emission and compare it with the existing H.E.S.S. data. We discuss the CR injection in the region through a spectral and morphology comparison. Finally, we discuss the values of the SNR rate and the diffusion parameters.

\section{CR injection: single or multiple accelerators?}

Several sky surveys have shown the presence of multiple impulsive accelerators throughout the GC. One of the major uncertainty for modelling theses sources is their rate as well as their spatial distribution. The GC harbors three compact and massive cluster: the Quintuplet (3-5 Myrs [13]), the Arches (2-3 Myrs [13]) and the central cluster surrounding SgrA ${ }^{\star}$ (4-6 Myrs [14]). Additionally, multiple observations [11] shown the presence of a high number of isolated massive star. Around $1 / 3$ of these sources are located outside of the three massive startburst cluster suggesting the evidence of isolated high mass stars formation in the GC. However these isolated stars could have been kicked out from the Arches or the Quintuplet cluster due to their dynamics [12]. The Arches cluster seems too young to have supernovae. In the following, we will test two spatial distributions. First, uniform distribution in the CMZ represented by a cylinder of radius $100 \mathrm{pc}$ and height $10 \mathrm{pc}$ and second a concentration of the SN in the Central and Quintuplet clusters with a uniform distribution for the numerous isolated stars. In both cases, SNs are modelled by impulsive CRs injections.

In addition, we investigate two scenarii of a single accelerator at the center: stationary for $\mathrm{SgrA}^{\star}$ and impulsive for SgrA East. For the sources, we consider a power-law CRs injection: $N_{o} E^{-a} \delta\left(r-r_{0}\right)$ for an impulsive source and $N_{o} E^{-a} H\left(r-r_{0}\right)$ for a continuous one. We assume a spectral index $a$ equal to 2 since this value is reproduced by the first order Fermi acceleration [19] for strong shocks. The efficiency of the CRs acceleration for the impulsive sources $\varepsilon$, is fixed to $10 \%$ of the kinetic energy released from a SN explosion $\left(E_{k}=10^{51} \mathrm{erg}[9]\right)$.

\section{CR propagation}

\subsection{Isotropic diffusion}

The CRs diffuse along the magnetic field lines when their Larmor radius is of the same order of magnitude that the wavelength magnetic wave $\lambda$. In this case, the particle is in resonance with the perturbation and then follows a random walk characterized by a mean free path $r_{m f p}\left(r_{m f p}=3 D / v\right)$ where $\mathrm{D}$ is the diffusion coefficient and $\mathrm{v}$ the particle's speed) before being scattered. For a sufficient period of time, the interaction between the charged particles and the magnetic inhomogeneities leads to their isotropization. The global CRs' propagation in the interstellar medium is then described by the simplified transport equation [20]. Neglecting the convection term, one has to resolve the very simple diffusion equation. The 3D Green function, obtained assuming a CR density equal to zero at $r=+\infty$, gives the solution for an impulsive accelerator. Integrating this solution gives the solution for a continuous source [20].

\subsection{Diffusion coefficient}

We assume a power-law diffusion coefficient: $\mathrm{D}=D_{o}(E / 10 \mathrm{GeV})^{d}$. The dependence $d$ of the diffusion coefficient depends on the turbulence spectrum of the $\mathrm{B}$ field. The detection of numerous 
non thermal radio filaments (NRFs), most of them oriented perpendicular to the Galactic Plane reveals the presence of a relatively strong and ordered magnetic field throughout the CMZ, approximately poloidal on average in the intercloud medium [17]. The magnetic field could reach $1 \mathrm{mG}$ inside the NRFs and could be much weaker in the general interstellar medium (ISM) (10 $\mu \mathrm{G}-50$ $\mu \mathrm{G}[18],[7])$.

A common value of the diffusion coefficient at $10 \mathrm{GeV}$ in the Galaxy is $\sim 10^{28} \mathrm{~cm}^{2} \mathrm{~s}^{-1}$ [20]. Some studies as [21] developed a model of strong turbulent magnetic field through which the hadrons must diffuse leading to a small value of the diffusion coefficient. This is why they ruled out the possibility of point source accelerators like the central black hole or the SNs along the disk and concluded with the necessity of stochastic particle acceleration. In the following, we will normalize the diffusion coefficient at $10 \mathrm{GeV}, D_{o}$, in order to have a value of $5 \times 10^{29} \mathrm{~cm}^{2} \mathrm{~s}^{-1}$ at 10 $\mathrm{TeV}$.

\subsection{Limit to the isotropic diffusion}

The mean free path $\left(r_{\mathrm{mfp}}\right)$ of the random walk characterizing the particle's diffusion along the magnetic field lines can reach $100 \mathrm{pc}$ for the highest CR energy simulated ( $1 \mathrm{PeV})$. For this energy, the mean free path is of the order of magnitude of the box. Therefore the diffusion approximation for this distance is wrong and we need to develop a proper approach to model the CR propagation in a ballistic regime and in particular the $\gamma$ rays flux produced since the particles are not isotropised any more. In this work, we adopt a very simple solution (in order to avoid a diffusion approximation) on these distances: we fix a constant value of the CR density for the distances $\mathrm{r}<$ $r_{o}=3 \times r_{m f p}$ equal to the CR density at $r=r_{o}$. This approach neglects the $\gamma$-ray emission from the non-isotropized CRs. The contribution of the latter should appear as a point source. This simple approach allows to properly take into account the diffuse emission under the source.

\section{4. $\gamma$ rays production}

\subsection{Matter distribution}

The Galactic Center presents a huge quantity of molecular matter in the CMZ that extends on $300 \mathrm{pc}$ in Galactic longitude and $100 \mathrm{pc}$ in latitude. This matter seems distributed in two molecular components: several dense molecular clouds and a widespread high-temperature and lower density diffuse component [8]. In order to model the 3D $\gamma$ rays distribution produced by the interaction of the CRs with the matter in the GC, one has to build a coherent 3D matter distribution.

The molecular line like CS or CO are the main tracers of the dense core of molecular gaz. However, since our knowledge of the true gas kinematics in the GC is very limited, the method used to convert the measured line of sight velocity into a radial distance are unreliable. A notable exception is the work of Sawada et al (2004) [15] who derived a face-on-map of the molecular gas without any kinematics assumption, relying only on two lines surveys (comparison between the CO 2.6-mm emission with the $\mathrm{OH} 18$-cm absorption). Supposing a total mass of $2 \times 10^{7} \mathrm{M}_{\text {sun }}$, we can convert their face on view intensity map in a density map assuming a same scaling factor in the whole CMZ. As no information on the $\mathrm{H}_{2}$ vertical gas distribution is provided, we assume an exponential decay along the Galactic latitude [16]. For the atomic gas, we used a $\mathrm{HI}$ mass around $10 \%$ of the $\mathrm{H} 2$ mass in the CMZ [16]. 


\section{$4.2 \gamma$-ray fluxes}

In order to model the total $\gamma$-ray flux created by the pp interaction, we summed over all the energies of the incident CRs the analytic shape found in [22] for this interaction with the CRs spectrum and the realistic matter distribution presented in the previous section. We simulate the injection and propagation of CRs ranging from $1 \mathrm{TeV}$ to $1 \mathrm{PeV}$ in a $3 \mathrm{D}$ box of size $500 p c \times$ $500 p c \times 50 p c$ centered on the GC. After integrating along the line of sight, we create 2D flux and spectral index maps in Galactic coordinates. In order to compare with the 1D Galactic longitude profil in [1], we sum the predicted emission along the latitude $\mathrm{b}\left(|b|<0.2^{\circ}\right)$.

\section{Results}

\subsection{Impulsive or stationary accelerator at the center?}

The Figure 1.a represents the profile along the Galactic longitude for a stationary source (modeling the black hole) and an impulsive injection $10 \mathrm{kyrs}$ ago (modeling the supernova SgrA east). The stationary profile is peaked on the source itself whereas the impulsive profile is peaked on the matter surrounding the source because the CRs have been emitted 10 kyrs ago and have already diffused, creating a flatter particle distribution. Considering the deficit of VHE emission beyond $100 \mathrm{pc}$ (in particular at $\mathrm{l}=1.3^{\circ}$ ) relative to the available target material, [1] concluded to injection 10 kyrs ago. However, the stationary $\gamma$-ray profile also drops at $|l|>1.3^{\circ}$ as the result of the integration of CR density with a more spread matter distribution along the line of sight (LOS) [15]. Therefore, the hypothesis of a stationary source at the GC capable to sustain a permanent injection of CRs is thus not to be excluded. Given the low statistics available so far, neither of these two hypothesis may still be excluded.

Figure 1.b compares the $\gamma$-ray energy spectra of the two models with the one described in [1]. We assumed Kolmogorov spectrum for the diffusion coefficient $(\mathrm{d}=0.3)$ in order to reproduce the hard spectrum observed with H.E.S.S $(\Gamma=2.3)$. For higher index $d$, the $\gamma$-ray spectrum for a stationary or an impulsive source will be too soft compared to the HESS data unless we assume harder intrinsic spectrum of the source around 1.8. The intrinsic power needed for the stationary source for CR acceleration in order to reproduce the total $\gamma$-ray flux observed with H.E.S.S. is around $3 \times 10^{38}$ $\mathrm{erg} \mathrm{s}^{-1}$ (significant fraction of the Bondi accretion power [5]). For a single impulsive injection with our propagation parameters, the assumed total mass of $2 \times 10^{7} \mathrm{M}_{\text {sun }}$ for the CMZ is not sufficient to reproduce the total flux.

In this section we have considered two kinds of injection for a unique source at the GC. However, in order to build a coherent picture, we have to study the impact of the recurrent explosions of massive stars leading to multiple CRs injection throughout the CMZ on the VHE flux.

\subsection{Impact of the SNRs in the CMZ}

In order to take into account individual SNRs distributed in the CMZ, we generate impulsive sources distributed according to a Poisson law of recurrence time $\tau=2500$ years (SN rate of 0.04 $\mathrm{yrs}^{-1}$ ). We keep only the SNs with an age $>1 \mathrm{kyr}$ (no younger SN has been observed in the GC) and $<100$ kyrs since for larger times the CR density becomes negligible. For the spatial distribution we consider the two models presented in section 2. We generate 50 temporal and spatial distributions. 


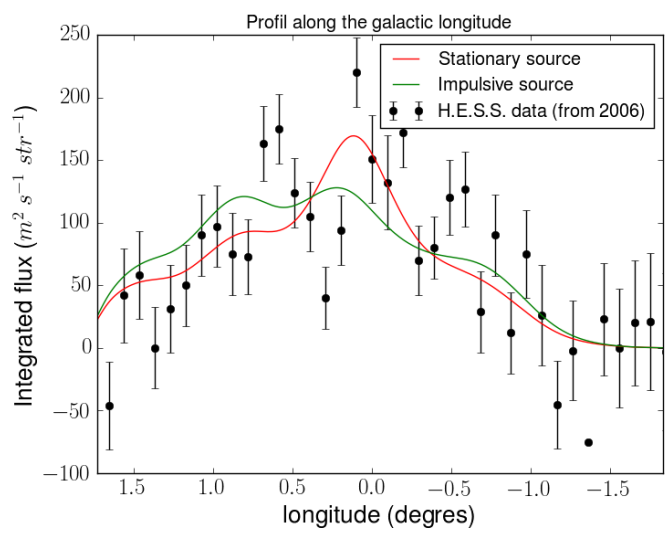

(a)

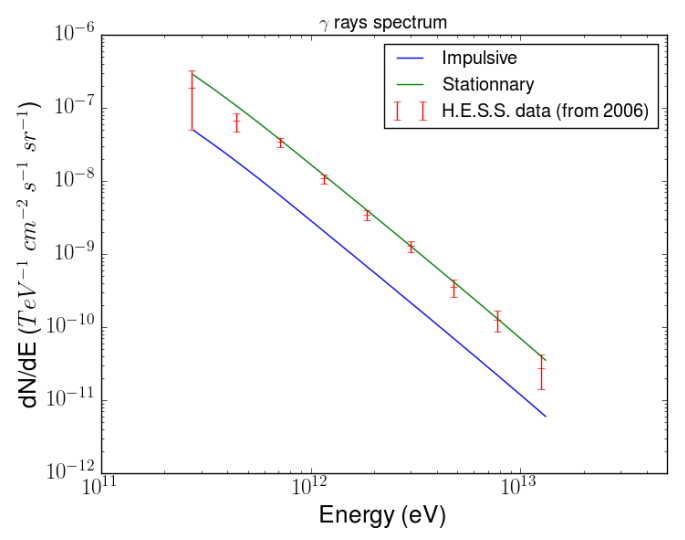

(b)

Figure 1: Modelling of VHE $\gamma$ profile along the Galactic longitude, after integrating along the line of sight and the Galactic latitude $\mathrm{b}$ for a stationary injection and for an impulsive injection $10 \mathrm{kyrs}$ ago at the center (a). The black points are the HESS data from [1]. The spectrum of the total VHE emission is visible in (c) for the impulsive and stationary injection as well as the spectrum obtained with the HESS data from [1]. The CRs injection is a power-law of index 2. The diffusion coefficient fallow a power-law: $\mathrm{D}=D_{10 T e V}(E / 10 \mathrm{GeV})^{d}$ with $\mathrm{D}_{10 /, \mathrm{TeV}}=5 \times 10^{29} \mathrm{~cm}^{2} \mathrm{~s}^{-1}$ and $\mathrm{d}=0.3$.

On Figure 2.a, we present the profile of the median of these fifty source configurations as well as the dispersion around this median for the two models. The profile is highly dependent on each SNs spatial and temporal distributions. Moreover, we see that taking into account a more realistic spatial distribution of SNR, with the presence of the clusters, makes the distribution peaked on the GC. The difference between the two models is even larger for lower diffusion coefficients.

The figure 2.b presents the total $\gamma$-ray energy spectrum in the GC produced by these multiple SNRs assuming Kolmogorov turbulences for the diffusion coefficient $(\mathrm{d}=0.3)$. The median spectrum as well as the dispersion around the median are shown. With the diffusion parameters used, the total resulting flux is larger than the one observed with HESS. Some studies consider the modeling of these distributed sources as a steady state model [7]. However especially for the high energy part of the spectrum, regarding the characteristic time between each explosion (around 2500 years), a stationary model can't be reached with the diffusion coefficient we are considering. The highest energetic particles have already diffused beyond the box between each explosion. However, in order to evacuate the excess of CRs caused by these multiple injection, they invoke the presence of a high speed wind (from 400 to $1000 \mathrm{~km} \mathrm{~s}^{-1}$ ) perpendicular to the Galactic plane. If we consider a typical scale in this direction for the advection of 50 pc height and the highest speed $v$ of their range, the characteristic time of this advection is $\tau_{a d} \approx H_{a d} / v \approx 50000$ years. The typical characteristic time for the diffusion on the same scale is $\tau_{\text {diffusion }} \approx H_{a d}{ }^{2} / 4 D$. In the case of $D_{10 T e V}=5 \times 10^{29} \mathrm{~cm}^{2} \mathrm{~s}^{-1}$ and $\mathrm{d}=0.3$ for the diffusion coefficient, $\tau_{\text {diffusion }} \approx 1000$ years at $1 \mathrm{TeV}$. Whatever the diffusion coefficient used, the wind does not solve the problem since the advection speed is not competitive with diffusion. However, for the low energies available by Fermi, the presence of this wind could help to evacuate the CRs since in this energy range $\tau_{\text {advvection }}<\tau_{\text {diffusion }}$.

In order to be compatible with the HESS flux, we have to consider a recurrence time of $10 \mathrm{kyrs}$ 


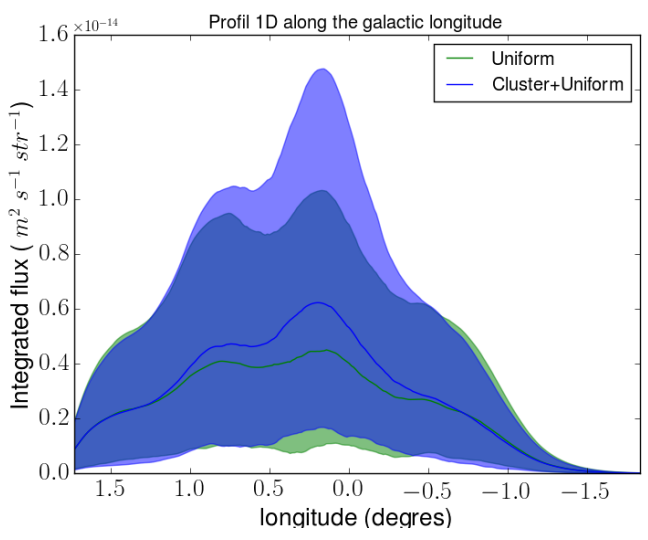

(a)

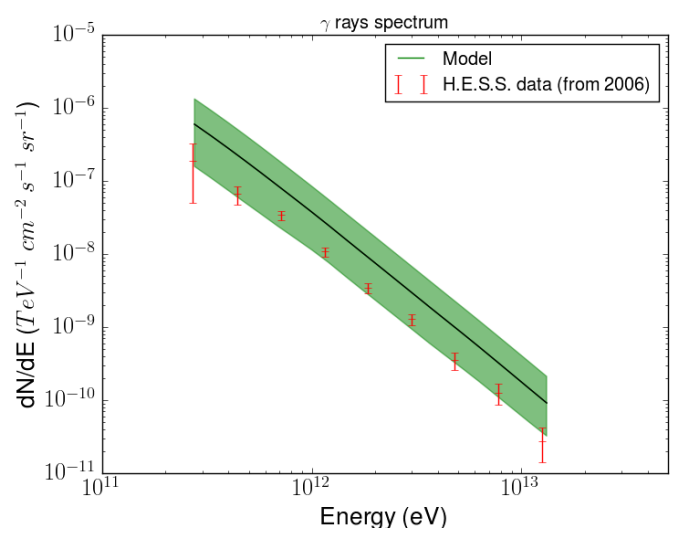

(b)

Figure 2: Modelling of the VHE $\gamma$ profile along the Galactic longitude, after integrating along the line of sight and the Galactic latitude b, produced by the SNs throughout the CMZ. We generate 100 configurations of SNs. We test two spatial distributions: an uniform distribution of the SNs in the GC (green) and a distribution of the SNs taking into account the two massive cluster: the Quintuplet and the central cluster (blue). The solid lines represent the median of these 100 draws and the colored regions the dispersion around this median. In (b) is shown the median of the spectrum of the 50 draws for a uniform distribution of SNs as well as the dispersion around the median. The red line is the HESS data spectrum. The CRs injection is a power-law of index 2. The diffusion coefficient fallow a power-law: $\mathrm{D}=D_{10 T e V}(E / 10 \mathrm{GeV})^{d}$ with $\mathrm{D}_{10 \mathrm{TeV}}=5 \times 10^{29} \mathrm{~cm}^{2} \mathrm{~s}^{-1}$ and $\mathrm{d}=0.3$.

that seems very high regarding the activity in the GC. In order to lower the total VHE flux using reasonable parameters on the $\mathrm{SN}$ rate and the diffusion, an explanation could be a low efficiency of CRs acceleration by the SNs in the GC. Regarding the very hot medium, the shock following the explosion could be weakly supersonic. In this case, the value of the intrinsic spectrum given by the first order of Fermi acceleration is larger than 2. With a softer injected spectrum $(a=2.2)$, a large recurrence time (>5000 yrs) is still required in order to be compatible with the H.E.S.S. data.

\section{Conclusion}

In order to study the origin of the VHE diffuse emission in the GC observed with H.E.S.S., we build a 3D model of CR injection and propagation with a realistic 3D gas distribution. We investigate two single source scenarios at the GC: an impulsive injection (SgrA East) and a stationary one $\left(\operatorname{SgrA}^{\star}\right)$. Since the VHE emission results of an integration along the LOS of the CR density with the matter, the deficit of emission at $|l|>1.3^{\circ}$ doesn't exclude a stationary model since it could only be due to a larger spread of matter along the LOS at these longitudes. However, a single source scenario can't be studied without taking into account the multiple CRs injection throughout the CMZ due to the numerous SNs observed in the GC. With reasonable propagation parameters, we found that assuming a characteristic value of the recurrence time for these sources around 2500 yrs, the $\gamma$-ray flux is much larger than the one observed with H.E.S.S. In order to solve this issue, we could consider either a higher recurrence time for the SNs but there is no observational evidence for this hypothesis or a lower efficiency for the $\mathrm{CR}$ acceleration considering the very hot medium 
where SNs are located. Another possibility, regarding the morphology of the magnetic field, could be a larger diffusion coefficient in particular in the direction perpendicular to the Galactic plane.

\section{References}

[1] F. Aharonian et al, 2006, Nature, 439, 695-698

[2] A. M. Ghez et al, 2008, ApJ, 689, 1044-1062

[3] F. K. Baganoff et al, 2003, ApJ, 591, 891-915

[4] D. P. Marrone et al, 2007, ApJ, 654, L57-L60

[5] Q. D. Wang et al, 2013, Science, 341, 981-983

[6] S. Liu et al, 2006, ApJ, 647, 1099-1105

[7] R. M. Crocker et al, 2011, MNRAS, 413, 763-788

[8] G. Dahmen et al, 1998, A\&A, 331, 959-976

[9] S. P. Reynolds, 2011, ApSS, 336, 257-262

[10] O. Macias et al, 2014, Arxiv e-prints

[11] J.C.Mauerhan et al, 2010, ApJ, 725, 188-199

[12] M. Habibi, et al, 2014, A\&A, 566, A6

[13] F.Najarro et al, 2004, ApJ, 611, L105-L108

[14] H. B. Liu et al, 2013, ApJ, 770, 44

[15] T. Sawada et al, 2004, MNRAS, 349, 1167-1178

[16] K.Ferrière, 2007, A\&A, 467, 611-627

[17] K. Ferrière et al, 2011, IAU Symposium, 271, 170-178

[18] T. N. LaRosa et al, 2005, ApJ, 626, L23-L27

[19] A.R.Bell, 1978, MNRAS, 182, 443-455

[20] V. S. Berezinskii, Astrophysics of cosmic rays, North Holland, 1990

[21] E. Wommer et al, 2008, MNRAS, 387, 987-997

[22] S. R. Kelner, 2006, Phys. Rev., 74, 034018 